\title{
A33 ELEVATED LEVELS OF CD5+ B CELLS IN SPONDYLOARTHRITIS PATIENTS
}

T Cantaert, Y Tiersma, C A Ambarus, J E Paramarta, P P Tak, L E De Rycke, D L Baeten Clinical Immunology and Rheumatology, Academic Medical Center, University of Amsterdam, The Netherlands

10.1136/ard.2010.129585g

Background We recently proposed that spondyloarthritis $(\mathrm{SpA})$ is characterised by primary alterations in the innate rather than the acquired immune system. However, SpA patients develop a strong IgM antinuclear antibody profile upon tumour necrosis factor blockade. The characteristics of these autoantibodies are compatible with natural antibodies originating from 'innate' B cells rather than with genuine, pathogenic autoantibodies. As in mice natural autoantibodies are produced by CD5 expressing peritoneal $\mathrm{B}$ cells responding to innate immune signals, we aimed to quantify and characterise the CD5 B cell subset in SpA.

Methods We collected peripheral blood mononuclear cells of $17 \mathrm{SpA}$ patients, 11 rheumatoid arthritis (RA) patients and 20 healthy controls. Subsets and activation markers were analysed using flow cytometry (FacsCanto, BD) and FlowJo software. CD5 and CD5 IgD+/CD27 B cells were sorted (FACSARIA, BD) ( $\mathrm{n}=\mathrm{HC}$ and $\mathrm{n}=\mathrm{SpA})$. Cells were cultured for $40 \mathrm{~h}$ with $\mathrm{T}$ cell dependent (interleukin 2 (IL-2)/CD40L/anti$\operatorname{IgM}$ ) or $\mathrm{T}$ cell independent (IL-2/CpG) stimuli and analysed for the expression of the activation marker CD80.

Results Total B cell numbers were similar between the three groups. Naive (IgD+/CD27), memory (IgD-/CD27) and marginal zone-like (IgD+/CD27) B cell populations were not different between the groups. Also subclassification of the $\mathrm{B}$ cells according to $\mathrm{CD} 38 / \mathrm{IgD}$ expression yielded no differences. In contrast, analysis of CD5 expression indicated an increased CD5 B cell population in SpA $(22.6 \%)$ versus HC $(13.6 \%)(p=0.048)$. No difference was observed between $\mathrm{HC}$ and RA patients. The difference in CD5 B cells between SpA and HC was most pronounced in the naive subset $(28.0 \%$ vs $12.8 \%, p=0.009)$ and the $\operatorname{IgD}+/$ CD27 subset $(18.4 \%$ vs $10.5 \%, p=0.006)$. Expression of the costimulatory molecules CD80 and CD86 was lowered in CD5 versus CD5 B cells both in SpA and HC $(p<0.05)$. In contrast, expression of the early activation marker CD69 was elevated in CD5 versus CD5 B cells both in SpA and HC $(p<0.05)$. After both $T$ cell dependent and $T$ cell independent stimulation, sorted CD5 naïve $B$ cells downregulated CD80 expression $(p<0.05)$ whereas CD5 B cells upregulated CD80 expression $(p<0.05)$. 
Conclusion These data indicate a significant increase of CD5 $B$ cells in SpA. Our preliminary data suggest that these cells could have an anergic phenotype. Further functional characterisation of this cell population is of particular interest in the context of a regulatory, IL-10 mediated function of these cells in the mice intestine and the association between SpA and inflammatory bowel disease in humans. 